\title{
Neoantigens Derived from Recurrently Mutated Genes as Potential Immunotherapy Targets for Gastric Cancer
}

\author{
Jie Zhou, ${ }^{1}$ Wenyi Zhao $\mathbb{D}^{2},{ }^{2}$ Jingcheng Wu, ${ }^{1}$ Jun Lu $\left(\mathbb{D},{ }^{3}\right.$ Yongfeng Ding, ${ }^{3}$ \\ Shanshan Wu, ${ }^{1}$ Haiyong Wang, ${ }^{3}$ Ding Ding, ${ }^{4,5}$ Fan Mo $\left(\mathbb{D},{ }^{4,6}\right.$ Zhan Zhou $\left(\mathbb{D},{ }^{1}\right.$ \\ Lisong Teng $\mathbb{D},{ }^{3}$ and Shuqing Chen $\mathbb{D}^{1}$
${ }^{1}$ Zhejiang Provincial Key Laboratory of Anti-Cancer Drug Research, College of Pharmaceutical Sciences, Zhejiang University, 866 Yuhangtang Road, Hangzhou, 310058, China
${ }^{2}$ Institute of Innovative Medicine, College of Pharmaceutical Sciences, Zhejiang University, Hangzhou 310058, China
${ }^{3}$ Department of Surgical Oncology, The First Affiliated Hospital, School of Medicine, Zhejiang University, 79 Qingchun Road, Hangzhou, 310003, China
${ }^{4}$ Hangzhou Neoantigen Bio-Tech Ltd., Co., 688 Road Binan, Hangzhou, 310051, China
${ }^{5}$ Formulation \& Analysis Lab, Hisun Pharma (Hangzhou) Co. Ltd., Xialian Village, Xukou Town, Fuyang, Hangzhou, 311404, China
${ }^{6}$ Vancouver Prostate Centre, University of British Columbia, 2660 Oak Street, Vancouver, BC, Canada V6H $3 Z 6$ \\ Correspondence should be addressed to Lisong Teng; lsteng@zju.edu.cn and Shuqing Chen; chenshuqing@zju.edu.cn
}

Received 25 January 2019; Accepted 12 May 2019; Published 13 June 2019

Guest Editor: Deborah Mukherji

Copyright (C) 2019 Jie Zhou et al. This is an open access article distributed under the Creative Commons Attribution License, which permits unrestricted use, distribution, and reproduction in any medium, provided the original work is properly cited.

\begin{abstract}
Neoantigens are optimal tumor-specific targets for T-cell based immunotherapy, especially for patients with "undruggable" mutated driver genes. T-cell immunotherapy can be a "universal" treatment for HLA genotype patients sharing same oncogenic mutations. To identify potential neoantigens for therapy in gastric cancer, 32 gastric cancer patients were enrolled in our study. Whole exome sequencing data from these patients was processed by TSNAD software to detect cancer somatic mutations and predict neoantigens. The somatic mutations between different patients suggested a high interpatient heterogeneity. $\mathrm{C}>\mathrm{A}$ and $\mathrm{C}>\mathrm{T}$ substitutions are common, suggesting an active nucleotide excision repair. The number of predicted neoantigens was significantly higher in patients at stage T1a compared to in patients at T2 or T4b. Six genes (PIK3CA, FAT4, BRCA2, GNAQ, LRP1B, and PREX2) were found as recurrently mutated driver genes in our study. Combining with highly frequent HLA alleles, several neoantigens derived from six recurrently mutated genes were considered as potential targets for further immunotherapy.
\end{abstract}

\section{Introduction}

Since the approval of trastuzumab as a treatment for HER2positive breast cancer in 1998 by the FDA [1], tumorassociated antigens such as CD molecules, VEGF, and EGFR have been actively targeted for drug development by the pharmaceutical industry [2-4]. The side effects of therapies based on monoclonal antibodies are mild and tolerable. However, when coupled with antibody-drug conjugates (ADC) or the chimeric antigen receptor T-cells (CAR-T) technology, the nonspecific and durable off-target cytotoxicity can be fatal for patients [5]. Therefore, the development of an optimal tumor-specific target that could differentiate tumor cells from normal tissues is essential.
Several studies have shown that targeting neoantigens in T-cell-based immunotherapy is a promising approach for treatment of lung adenocarcinomas [6], leukemia [7], and melanoma $[8,9]$. Cancer is initialized by somatic driver mutations and other genetic instabilities, which are the molecular basis of the carcinogenesis process. In particular, point mutations are directly involved in essential cellular activities and functions, such as proliferation, apoptosis, and tumorigenesis. Mutant proteins are also processed by the intracellular repair system through ubiquitination and hydrolysis in the proteasome. Hydrolyzed peptides (length of 8-11 amino acids) are bonded with class I major histocompatibility complex (MHC) molecules and are presented on the cell 
surface as tumor-specific neoantigens, which are recognized by T-cells, provoking an immune response.

Gastric cancer (GC) is the third leading cause of cancer mortality in world. It is a common cancer prevalent in Eastern Asia, Central and Eastern Europe, and South America. The prognosis remains poor with a 5-year overall survival rate at $30.4 \%[10,11]$. Besides traditional chemotherapy agents, only trastuzumab, ramucirumab, and apatinib have been approved for advanced or metastatic GC. Systematic molecular profiling of GC on 595 patients by the Cancer Genome Atlas (TCGA) [12] and Asian Cancer Research group (ACRG) [13] shows that CG are highly heterogenous, exhibiting high chromosomal instability, hypermethylation, and mutation burden. Based on its molecular characteristics, the identification of neoantigens against recurrently mutated oncogenes is feasible, using current next-generation sequencing (NGS) platforms and bioinformatic analysis pipeline.

Previous studies have used genomic data from the TCGA, Foundation Medicine Adult Cancer Clinical Dataset (FM$\mathrm{AD}$ ), and their own cohorts to characterize neoantigens and their association with genetic alteration or with survival [1417]. However, these studies did not focus on neoantigen profiling for gastric cancer patients. We analyzed the characteristics of somatic mutations and neoantigens, especially their correlation with clinical features of patients. The important neoantigens and their associated oncogenes shared by several patients were chosen with the goal of further developing $\mathrm{T}$ cell-based immunotherapy such as vaccines for patients. The work presented here collected tumor tissues and peripheral blood samples from 32 gastric cancer patients. The whole exome sequencing was performed on Illumina Hiseq4000 sequencing system. An in-house developed integrated software "Tumor-Specific Neo-Antigen Detector" (TSNAD) [18] was used to predict neoantigens.

\section{Materials and Methods}

2.1. Patients. Fresh or FFPE-embedded primary tumor tissues and paired peripheral blood were collected from 32 gastric cancer patients during the period from August 12, 2016, to March 14, 2017. Among the 32 gastric patients, 11 were female patients and 4 were below 45 years of age. Of these, 2 were T1a, 6 were T2, 6 were T4a, and 18 were T4b cases, respectively. Detailed information of these samples is listed in Table 1. The enrolment of human subjects in this study was done after informed consent forms were signed. Written consent for the collection and use of tissues for research purposes has been obtained, with ethical approval from Research Ethics Committee of the First Affiliated Hospital, Zhejiang University School of Medicine, China. All methods reported in our study were performed in accordance with the relevant guidelines and regulations.

2.2. Whole Exome Sequencing. DNA was extracted from the tumor tissues and peripheral blood using AxyPrep Blood Genomic DNA Kit and AxyPrep Multisource Genome DNA Kit. Exomes were captured from $750 \mathrm{ng}$ of genomic DNA per sample using the Agilent SureSelect Human All Exon V5 Kit (Agilent Technologies) according to the manufacturer's instructions. Paired-end multiplex sequencing was then performed on the Illumina HiSeq4000 sequencing platform. On average, the sequencing depth was $86 \times$ per sample with standard deviation $\pm 46 \times$.

\subsection{Pipeline for Somatic Mutation Analysis, HLA Genotyping,} and Neoantigen Prediction. The raw data was processed by integrated software TSNAD (available on http://github.com/ jiujiezz/TSNAD) [18]. This software was developed by our laboratory with a graphical user interface, which combines the necessary algorithms to identify cancer somatic mutations, determine HLA genotyping, and predict neoantigens. TSNAD can identify cancer somatic mutations following the best practices of the genome analysis toolkit (GATK) from the genome/exome sequencing data of tumor-normal pairs and also determine HLA genotyping by SOAP-HLA [19]. Then, TSNAD invokes NetMHCpan [20] to predict neoantigens which can bind to class I MHC molecules. Besides, TSNAD can also identify germline mutations.

2.4. Statistical Analysis. The statistical analyses were performed using $\mathrm{R}$ software and Wilcoxon rank-sum test was used to determine the significance. The significance was defined when $\mathrm{p}<0.05$.

\section{Results and Discussion}

3.1. Intrapatient Heterogeneity of Somatic Mutations and Neoantigen Number. The somatic mutation analysis and neoantigen prediction of 32 gastric cancer patients were directly performed by TSNAD from whole exome sequencing raw data. The numbers of nonsynonymous mutations, indels, and neoantigens were listed in Table 1 . In total 7,432 somatic missense mutations, 658 indels, and 12,929 neoantigens were filtered by the software for 32 patients. The median number is 138 for missense mutation (median tumor mutation burden, median TMB $=4.6$ mutations $/ \mathrm{Mb}$ ), 14 for indels, and 202 for neoantigens (see Figures 1(a) and 1(c)). The fitting curve revealed a laniary positive correlation between missense number and predicted neoantigens $\left(\mathrm{R}^{2}=0.8845\right.$, see Figure 1(b)). According to three gastric cancer projects in ICGC Project (GACA-CN, GACA-JP, and STAD-US), the $\mathrm{TMB}$ of Chinese gastric cancer patients (median $\mathrm{TMB}=$ 8.467 mutations $/ \mathrm{Mb}$ ) is greater than the Japanese (median $\mathrm{TMB}=6.467$ mutations $/ \mathrm{Mb}$ ) and American (median TMB $=5.3$ mutations $/ \mathrm{Mb}$ ). The difference in the tumor mutation burden between our cohort and GACA-CN is caused by the high heterogeneity of gastric cancer and the limited small sample size of our cohort.

However, there is a large variation in the number of somatic mutations between the patients studied, indicating significant molecular heterogeneity within gastric cancer. $71.9 \%(23 / 32)$ of patients had less than 200 missense mutations in coding regions, but there were two female patients (6.25\%) who surprisingly had more than 1300 mutations (high tumor mutation burden, TMB > 40 mutations/Mb) without any experimental bias. The difference of predicted neoantigen number is similar to mutation number. The number of indels varies from 5 to 104 . The majority of patients 
TABLE 1: The characteristics of patients and the number of mutations/neoantigens in 32 gastric cancer patients.

\begin{tabular}{|c|c|c|c|c|c|c|c|}
\hline Sample ID & Gender & Age & TNM & $\mathrm{T}$ & Number of missense mutations & Number of indels & Number of neoantigens \\
\hline NAG1606003 & $\mathrm{M}$ & 71 & T4bN2M1 & $\mathrm{T} 4 \mathrm{~b}$ & 190 & 11 & 323 \\
\hline NAG1606008 & M & 80 & T4bN2M0 & $\mathrm{T} 4 \mathrm{~b}$ & 158 & 35 & 208 \\
\hline NAG1606009 & M & 62 & T4bN1M0 & $\mathrm{T} 4 \mathrm{~b}$ & 109 & 7 & 133 \\
\hline NAG1606010 & M & 68 & T2N0M0 & $\mathrm{T} 2$ & 182 & 20 & 134 \\
\hline NAG1606011 & $\mathrm{F}$ & 45 & T4aN3bM0 & $\mathrm{T} 4 \mathrm{a}$ & 1336 & 59 & 2121 \\
\hline S0616092301 & $\mathrm{F}$ & 54 & T4bN1M0 & $\mathrm{T} 4 \mathrm{~b}$ & 9 & 5 & 4 \\
\hline S0616092302 & $\mathrm{F}$ & 58 & T4aN1M0 & $\mathrm{T} 4 \mathrm{a}$ & 145 & 31 & 242 \\
\hline S0616092903 & M & 61 & T2N1M0 & $\mathrm{T} 2$ & 115 & 8 & 190 \\
\hline S0616092904 & M & 76 & T4bN3aM0 & $\mathrm{T} 4 \mathrm{~b}$ & 217 & 12 & 297 \\
\hline S0616093001 & M & 67 & T4aN1M & $\mathrm{T} 4 \mathrm{a}$ & 233 & 24 & 223 \\
\hline S0616101201 & $\mathrm{M}$ & 65 & T4aN0M0 & $\mathrm{T} 4 \mathrm{a}$ & 102 & 17 & 134 \\
\hline S0616101301 & $\mathrm{M}$ & 38 & T4bN2M0 & $\mathrm{T} 4 \mathrm{~b}$ & 77 & 9 & 37 \\
\hline S0616101302 & $\mathrm{M}$ & 73 & T4bN1M0 & $\mathrm{T} 4 \mathrm{~b}$ & 124 & 17 & 351 \\
\hline S0616101901 & $\mathrm{F}$ & 52 & $\mathrm{~T} 4 \mathrm{aN} 2 \mathrm{M} 0$ & $\mathrm{~T} 4 \mathrm{a}$ & 63 & 12 & 83 \\
\hline S0616102501 & $\mathrm{F}$ & 57 & $\mathrm{~T} 2 \mathrm{~N} 2 \mathrm{M} 0$ & $\mathrm{~T} 2$ & 128 & 16 & 220 \\
\hline S0616102502 & M & 66 & TlaN1M0 & Tla & 177 & 9 & 666 \\
\hline S0616102601 & $\mathrm{F}$ & 62 & $\mathrm{~T} 4 \mathrm{bN} 2 \mathrm{M} 0$ & $\mathrm{~T} 4 \mathrm{~b}$ & 78 & 9 & 70 \\
\hline S0616102602 & $\mathrm{M}$ & 49 & $\mathrm{~T} 4 \mathrm{bN} 2 \mathrm{M} 0$ & $\mathrm{~T} 4 \mathrm{~b}$ & 150 & 14 & 206 \\
\hline S0616102801 & $\mathrm{F}$ & 50 & T4bNM0 & $\mathrm{T} 4 \mathrm{~b}$ & 1672 & 104 & 4896 \\
\hline S0616110101 & M & 64 & T4bN3aM0 & $\mathrm{T} 4 \mathrm{~b}$ & 331 & 43 & 313 \\
\hline S0616110401 & $\mathrm{F}$ & 41 & T4bN3aM0 & $\mathrm{T} 4 \mathrm{~b}$ & 89 & 9 & 57 \\
\hline S0616110801 & $\mathrm{F}$ & 51 & T4Bn3aM0 & $\mathrm{T} 4 \mathrm{~b}$ & 17 & 14 & 32 \\
\hline S0616110901 & $\mathrm{F}$ & 50 & T2N3aM0 & $\mathrm{T} 2$ & 153 & 6 & 198 \\
\hline S0616111101 & M & 59 & TlaN1M0 & Tla & 215 & 8 & 334 \\
\hline S0616111501 & $\mathrm{M}$ & 62 & T4bN1M0 & $\mathrm{T} 4 \mathrm{~b}$ & 127 & 7 & 186 \\
\hline S0616111801 & $\mathrm{F}$ & 60 & T4bN3aM0 & $\mathrm{T} 4 \mathrm{~b}$ & 70 & 9 & 85 \\
\hline S0616112401 & $\mathrm{M}$ & 58 & T2N3bM0 & $\mathrm{T} 2$ & 131 & 7 & 211 \\
\hline S0616112901 & $\mathrm{M}$ & 54 & T4bN1M0 & $\mathrm{T} 4 \mathrm{~b}$ & 78 & 22 & 122 \\
\hline S0616121301 & $\mathrm{M}$ & 42 & T4bN3aM0 & $\mathrm{T} 4 \mathrm{~b}$ & 125 & 9 & 127 \\
\hline S0617010301 & $\mathrm{M}$ & 64 & T2N2M0 & $\mathrm{T} 2$ & 258 & 16 & 116 \\
\hline S0617020601 & M & 60 & T2N1M0 & $\mathrm{T} 2$ & 308 & 39 & 306 \\
\hline S0617020701 & $\mathrm{M}$ & 63 & T4bN2M1 & $\mathrm{T} 4 \mathrm{~b}$ & 427 & 50 & 327 \\
\hline
\end{tabular}

had less than 300 neoantigens. The great number of missense mutations results in a great number of predicted neoantigens. The same two female patients had maximum neoantigens, 2,121 and 4,896, respectively.

Patient S0616092301 only had nine missense mutations and four predicted neoantigens, while patient S0616102801 had 1,672 missense mutations and 4,896 neoantigens. Despite great difference of mutation burden between minimum and maximum, these are two nonsmoking female patients both at their fifties diagnosed with T4b stage of gastric cancer.

3.2. $C>A$ and $C>T$ Substitutions Are Major Mutation Types in Gastric Cancer. We have observed an average of 232 nonsynonymous mutations in GC. In comparison with a study from Bi et al., we found that nonsynonymous mutation counts in GC were significantly higher than meningioma, thyroid cancer, pituitary adenoma, craniopharyngioma, breast cancer, and glioblastoma [21]. Our result was also in accordance with mutational signatures generated by Alexandrov et al. [22, 23].
We analyzed nucleotide substitution types of 7,432 missense mutations and found that $60.47 \%$ of missense mutations are transversions and $39.53 \%$ of substitutions are transitions. Individual types of substitution were presented at the bottom of Figure 2. On average, the percentage of $\mathrm{C}>\mathrm{A}$ type is $32.18 \%, 27.24 \%$ for $\mathrm{C}>\mathrm{T}, 12.51 \%$ for $\mathrm{T}>\mathrm{G}, 12.29 \%$ for $\mathrm{T}>\mathrm{C}$, 9.89\% for $\mathrm{C}>\mathrm{G}$, and $5.89 \%$ for $\mathrm{T}>\mathrm{A}$. $\mathrm{C}>\mathrm{A}$ and $\mathrm{C}>\mathrm{T}$ became the major substitution types in missense somatic mutations.

COSMIC has provided a set of 30 mutational signatures based on a large-scale analysis across nearly 40 human cancer types. 11 of 30 mutational signatures are reported related to gastric cancer. The dominant prevalence of $\mathrm{C}>\mathrm{A}$ and $\mathrm{C}>\mathrm{T}$ suggested a hyperactive deamination and transcribed strand bias during transcription-coupled nucleotide excision repair in gastric cancer.

3.3. Twelve Recurrently Mutated Genes in Gastric Cancer. The total 7,432 missense mutations were distributed in 4,451 genes. Firstly, we filtered the genes that had been discovered 


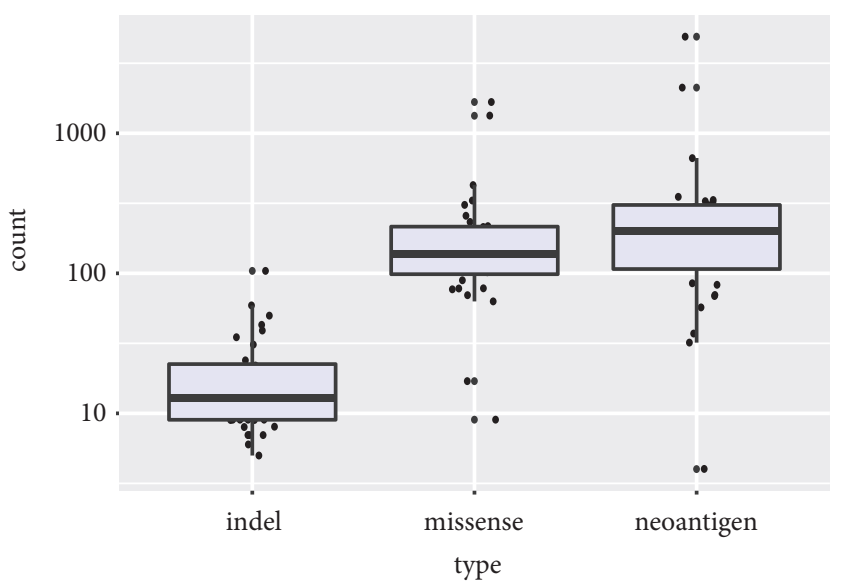

(a)

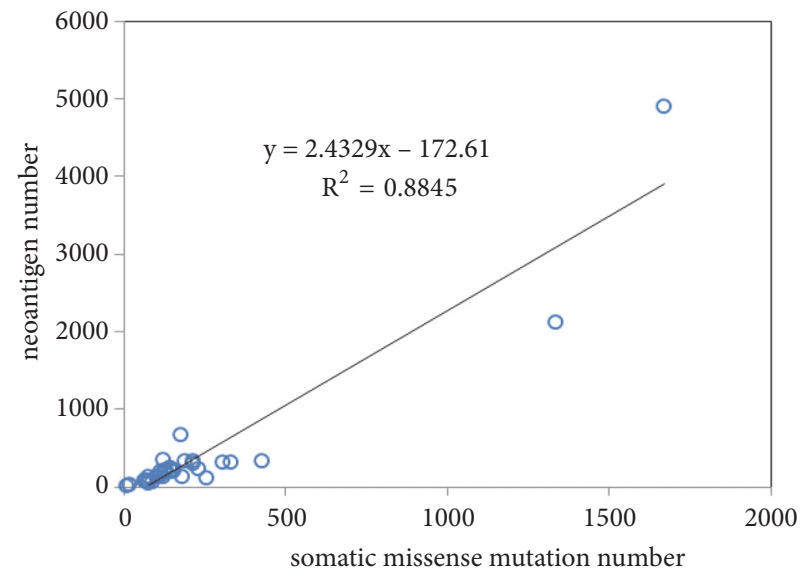

(b)

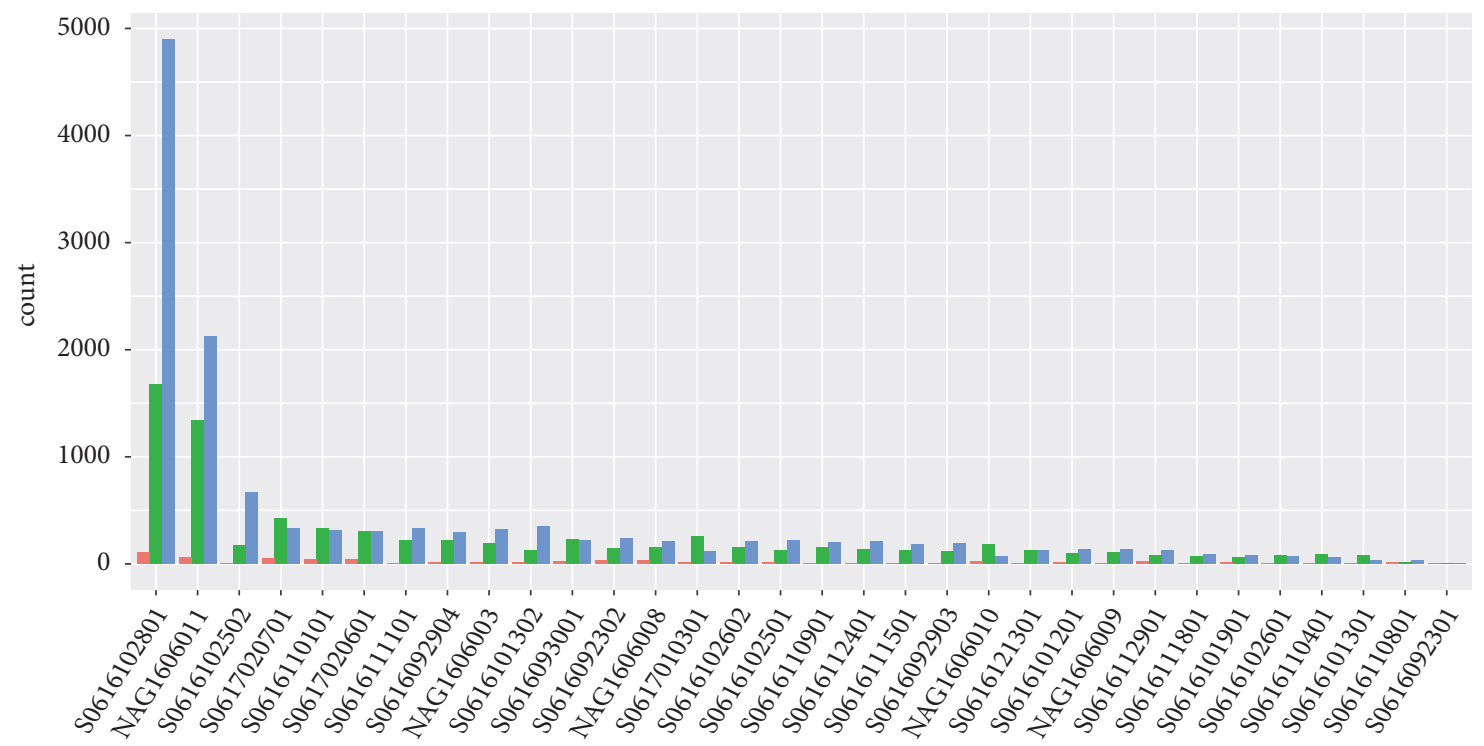

sample ID

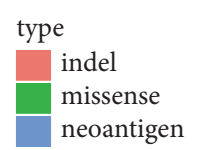

(c)

FIGURE 1: The overall number of somatic mutations and neoantigens. (a) The boxplot shows the numbers of indels, missense mutations, and predicted neoantigens. (b) The fitted curve between the number of somatic mutations and neoantigens, $R^{2}=0.8845$. (c) The histogram of numbers of indels, missense mutations, and neoantigens for each patient.

to be mutated at least in three patients. The number of genes decreased to 232 (see Additional file 1: Table S1). The upper part of Figure 2 presented a heat map of 232 mutant genes distributed in 32 patients. The most recurrently mutated gene was MUC4 with an occurrence of $93.75 \%$ in this study and more than 8 nonsynonymous mutations per patient. Mucin 4 is an integral membrane glycoprotein. As major constituents of mucus, Mucin 4 plays important roles in the protection of epithelial cells in the colon, cervix, and trachea. Silencing or reduced expression of MUC4 is associated with proliferation of pancreatic carcinoma cell line [24] and poor prognosis in renal cell carcinoma and breast carcinogenesis $[25,26]$. However, MUC4 has not been yet considered as a cancerrelated gene in COSMIC.

We then matched these 232 recurrent genes to the Cancer Gene Census; only 12 genes were filtered as essential tumorrelated genes: TP53 ( $\mathrm{n}=9,28.13 \%), L R P 1 B(\mathrm{n}=8,25 \%)$, PREX2 $(\mathrm{n}=6,18.75 \%)$, NRG1 $(\mathrm{n}=4,12.5 \%)$, PCM1 $(\mathrm{n}=3,9.38 \%)$, BRCA2 $(\mathrm{n}=3,9.38 \%)$, NOTCH1 $(\mathrm{n}=3,9.38 \%)$, USP6 $(\mathrm{n}=3$, 9.38\%), PIK3CA (n=3,9.38\%), GNAQ (n=3, 9.38\%), FAT4 $(\mathrm{n}=3,9.38 \%)$, and CDH1 $(\mathrm{n}=3,9.38 \%)$. PREX2 and PIK3CA are reported as famous oncogenes. By inhibition of PTEN 


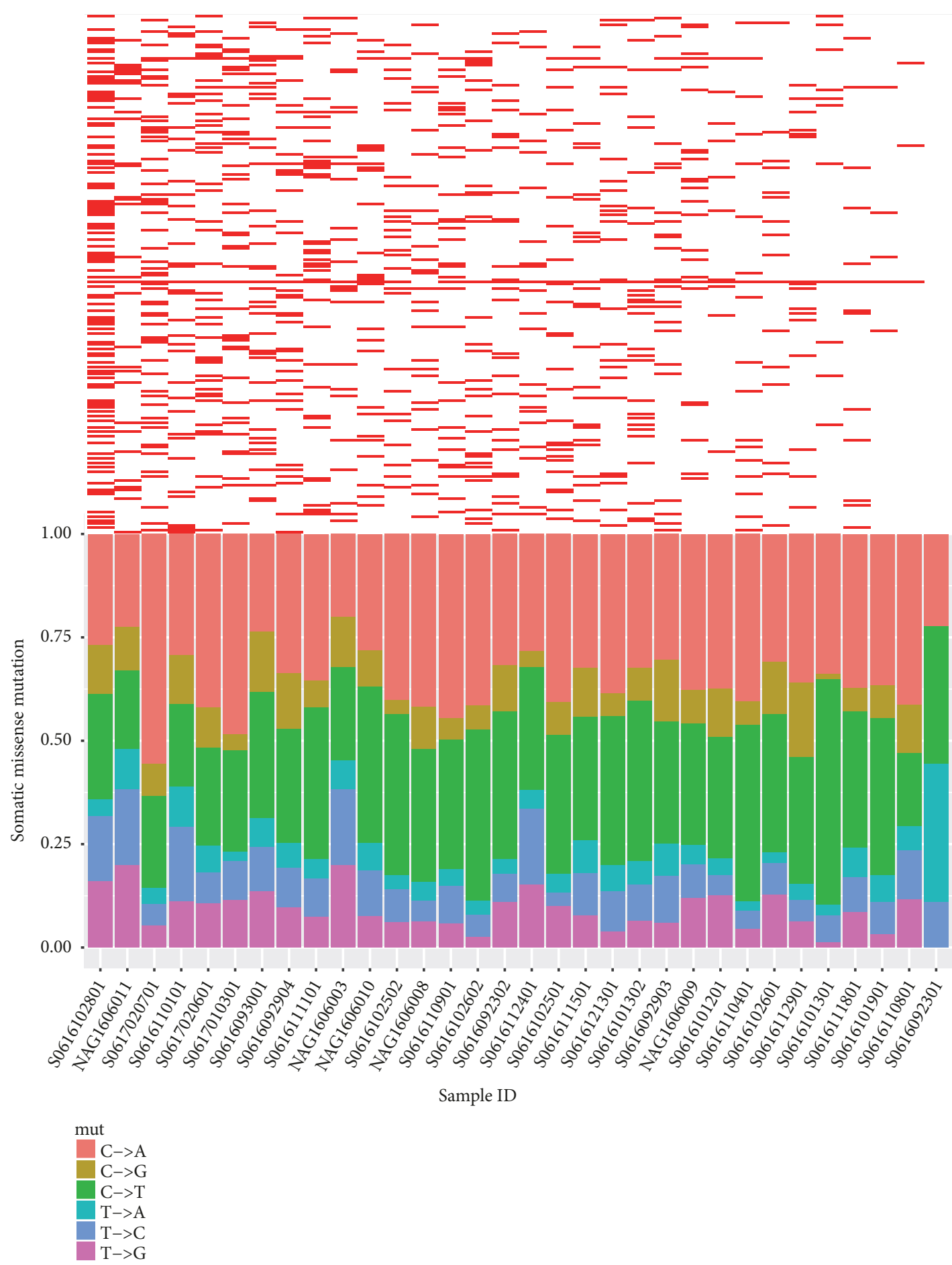

FIGURE 2: Mutational characteristics for 32 gastric cancers: upper: heat map for distribution of 232 recurrently mutated genes in 32 GC patients; bottom: percentage of missense substitution types; $\mathrm{C}>\mathrm{A}$ and $\mathrm{C}>\mathrm{T}$ are the major types in $32 \mathrm{GC}$ patients.

activity, PREX2 interacts with PIK3CA signaling pathway. In the four gastric cancer subtypes classified by TCGA, PIK3CA mutations occur at a frequency between $3 \%$ and $42 \%$ [12]. TP53, NOTCH1, FAT4, CDH1, and BRCA2 are tumor suppressor genes (TSG). TP53 somatic mutations were observed in $71 \%$ of chromosomal instability (CIN) subtypes and $\mathrm{CDH1}$ mutations were enriched in $37 \%$ of genomically stable (GS) subtype [12].

3.4. Neoantigen Profiling of 32 Gastric Cancer Patients Revealed Significant Differences between Stages. We tried to study the feature of neoantigens' number associated with 


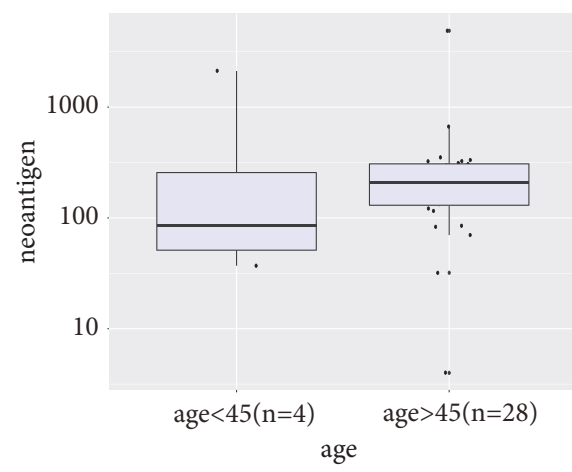

(a)

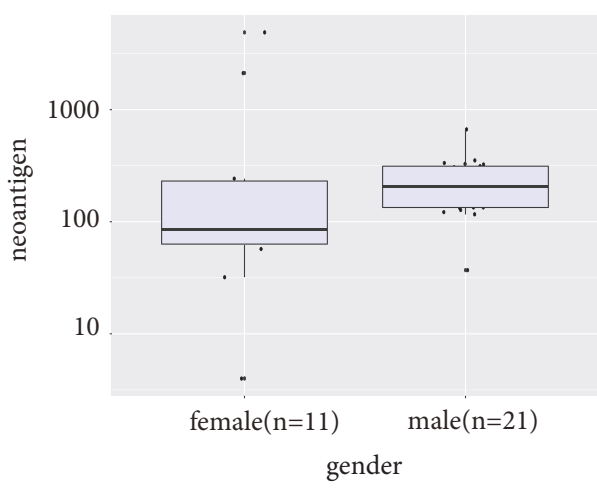

(b)

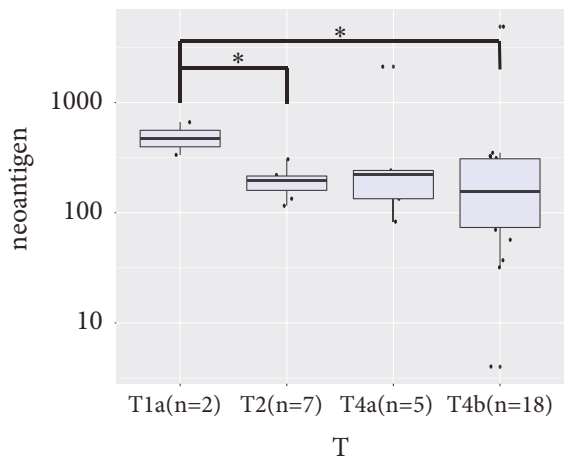

(c)

FIGURE 3: The boxplot of neoantigens' number subgrouping by age, gender, and stages. (a) The patients below 45 years $(n=4)$ and patients above 45 years $(n=28)$ showed no significant difference $(\mathrm{p}=0.1525)$. (b) The female patients $(\mathrm{n}=11)$ and male patients $(\mathrm{n}=21)$ showed no significant difference ( $p=0.0795)$. (c) The number of neoantigens differed among Tla $(n=2)$, T2 $(n=7)$, T4a $(n=5)$, and T4b ( $=18)$; the difference was significant between Tla and T2 $(\mathrm{p}=0.0202)$ and between Tla and T4b $(\mathrm{p}=0.0294)$.

patients' clinical characteristics (see Figure 3). Our study enrolled 11 female and 21 male patients; the median number of neoantigens for male patients was higher than that for female patients. The patients below 45 years of age $(n=4)$ had less predicted neoantigens than those elder than 45 years $(n=28)$. However, the difference was not statistically significant.

GC patients are usually diagnosed at more advanced stages of cancer progression. Of the 32 patients studied, 23 were diagnosed at T4 stage. Only 2 patients were diagnosed at T1 stage and 7 patients at T2 stage. Unexpectedly, the number of neoantigens was significantly higher in Tla than in $\mathrm{T} 2(\mathrm{p}=0.02)$ and $\mathrm{T} 4 \mathrm{~b}(\mathrm{p}=0.03)$ respectively. The reason why neoantigens are less common at later stages might be due to the enrichment of dominant malignant subclones as the tumor progressed. We continued to enroll early-stage patients to see that this pattern is statistically significant.

\subsection{Six Recurrently Mutated Genes Encoding Neoantigens} Predicted to Be Potential Targets against GC. Based on the HLA genotype (see Additional file 2: Table S2) and missense mutations of 32 patients, TSNAD performed neoantigen profiling according to the affinity of mutant peptides and HLA class I molecules. The alleles HLA-A $* 11: 01(46.9 \%)$, HLA-C $* 01: 02$ (37.5\%), HLA-A $* 03: 01$ (25\%), HLA-A $* 24: 02$ (25\%), and HLA-B*40:01 (21.9\%) were the most frequent alleles in our study. The diversity of HLA was similar to the research carried out by Gourraud et al. on 90 Han Chinese from Beijing dataset of the 1000 Genomes Project [27].

From 12,929 predicted neoantigens, we focused on the recurrently mutated genes encoding neoantigens that were present in at least 3 patients. 54 genes were filtered, and a heat map was made to visualize the distribution (see Figure 4). Then we matched 54 genes to the cancer Gene Census. PIK3CA, FAT4, BRCA2, GNAQ, LRP1B, and PREX2 were found as genes highly associated with cancer development.

The detailed mutations, HLA alleles, and sequences of 139 neoantigens derived from these six mutated genes were listed in Table S3 (see Additional file 3). The missense mutations found in PIK3CA, FAT4, BRCA2, LRP1B, and PREX2 were not recurrent in our study and the sequences of predicted neoantigens were unique. The amino acid change of T96S in GNAQ was the only same mutation found in three patients and the mutant peptides were predicted to bind to HLA$\mathrm{A} * 02: 01, \mathrm{HLA}-\mathrm{A} * 03: 01$, and HLA-A $* 11: 01$ alleles showing a strong binding ability (affinity IC50 $<100 \mathrm{nM}$ ). Unfortunately, the predicted neoantigen sequences for T96S in GNAQ were not identical for patients because of different individual HLAA genotype.

Due to the small size of enrolment, we used the mutation frequency published by the ICGC Project as a reference. We 


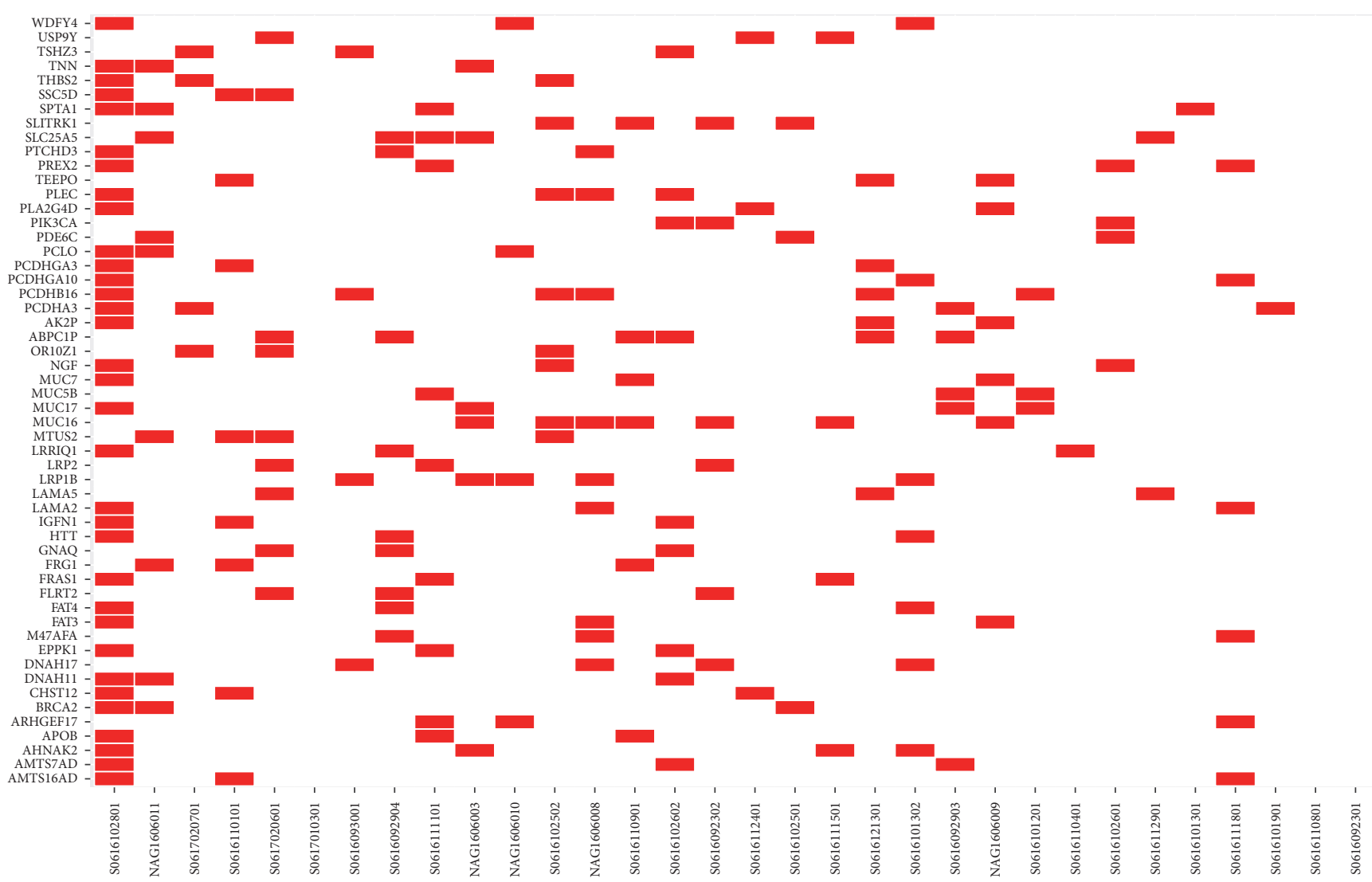

FIGURE 4: Heat map of individual distribution of 54 recurrently mutated genes which encode neoantigens.

found that mutations S37L $(1 / 12,198)$ and N289H $(3 / 12,198)$ in BRCA2, Q453L $(5 / 12,198)$ and A807V $(6 / 12,198)$ in FAT4, T96S $(10 / 12,198)$ in GNAQ, and H1047Y $(8 / 12,198)$ and V344M $(5 / 12,198)$ in PIK3CA have been already reported by the ICGC dataset (see Table 2) despite a very low frequency of occurrence. PIK3CA and its signaling pathway have been widely studied in many cancers. H1047 is a hotspot mutation in PIK3CA. Alternation from histidine to arginine has 281 recurrences of 12,198 donors in ICGC database. Our patient S0616102601 had the same position mutation but with an alteration from histidine to tyrosine. Only 1 donor from the ICGC gastric cancer dataset exhibited an identical mutation. In addition, we found two germline mutations in BRCA2 (A2466V and N372H). In ClinVar, A2466V and N372H are considered as benign mutations in familial breast cancer. $\mathrm{N} 372 \mathrm{H}$ is also annotated as a variant of unknown significance in Online Mendelian Inheritance in Man (OMIM).

The development of effective therapies against cancer has been a longstanding goal for many decades. Improvements in anticancer therapies rely on deeper understanding of the oncology and molecular basis of cancer. Recent positive developments in immunotherapy targeting neoantigens show promise as an effective method to treat cancer.

Neoantigens-associated immunotherapy could be a feasible strategy for treating malignancy with somatic mutations in driver genes encoding intracellular protein such as KRAS. KRAS has been considered as "undruggable" because of lack of binding pocket for the past 30 years. The famous amino acid alternation of Gly12 (G12V, G12C, or G12D) of KRAS protein is involved in $60-70 \%$ of pancreatic cancers and 20 $30 \%$ of colorectal cancers [28]. Work from Rosenberg and Tran has reported impressive response from G12D-positive metastatic colorectal cancer patients when treated with autologous T-cell therapy using the HLA-C*08:02 allele [29]. Of the 7 patients treated, 6 patients were reported to respond positively and are currently in remission. Mutations on KRAS are also an important cause for EGFR-targeted tyrosine kinase inhibitor (TKI) drug resistance. Moreover, the mutant peptides derived from V600E in BRAF [30, 31] or T790M in EGFR [32] were reported as binding with HLA-A $* 02: 01$ and presented as neoantigens. Therefore, neoantigens-associated immunotherapy could be employed to treat cancers exhibiting common drug resistance mutations.

In the top 20 recurrently mutated genes of three ICGC projects (GACA-CN, GACA-JP, and STAD-US), seven genes were shared between Chinese and Japanese gastric cancer patients. This included TP53, LRP1B, KMT2C, KMT2D, $A P C, G R M 3$, and SETD1B. Meanwhile, there are eight genes (TP53, LRP1B, KMT2C, KMT2D, APC, ATRX, NOTCH1, and SETBP1) shared between Chinese and American patients. Nine genes (NKX2-1, PTPN11, ATM, COLSA1, EPHA3, FOXO1, MSN, MYH11, and TSC1) are unique to the Chinese patients. Our results revealed 12 cancer-related genes (TP53, LRP1B, PREX2, NRG1, PCM1, BRCA2, NOTCH1, USP6, 
TABLE 2: The list of mutations of six recurrently mutated genes, mutation position, number of patients affected, corresponding patient HLA allele, number of predicted neoantigens, and number of patients affected in ICGC project.

\begin{tabular}{|c|c|c|c|c|c|}
\hline Gene & $\begin{array}{c}\text { Mutation in } \\
\text { Protein Position } \\
\end{array}$ & $\begin{array}{l}\text { Number of patients affected } \\
\text { in this study (total 32) }\end{array}$ & Patient HLA Allele & $\begin{array}{c}\text { Number of predicted } \\
\text { neoantigens }\end{array}$ & $\begin{array}{c}\text { Number of patients affected } \\
\text { in ICGC (total 12,198) }\end{array}$ \\
\hline \multirow{11}{*}{$B R C A 2$} & & & B58:01 & & \\
\hline & $\mathrm{I} 27 \mathrm{~V}$ & 1 & C03:02 & 6 & 0 \\
\hline & & & C08:01 & & \\
\hline & & & B15:02 & & \\
\hline & S37L & 1 & B58:01 & 6 & 1 \\
\hline & & & C03:02 & & \\
\hline & V144F & 1 & $\begin{array}{c}\text { B15:02 } \\
\text { C03:02 }\end{array}$ & 5 & 0 \\
\hline & Q147R & 1 & A33:03 & 2 & 0 \\
\hline & D156E & 1 & A11:01 & 1 & 0 \\
\hline & $\mathrm{N} 289 \mathrm{H}$ & 1 & $\begin{array}{l}\text { A02:01 } \\
\text { C03:02 }\end{array}$ & 5 & 3 \\
\hline & $\mathrm{T} 2542 \mathrm{M}$ & 1 & C14:02 & 2 & 0 \\
\hline \multirow{6}{*}{ FAT4 } & Q453L & 1 & $\begin{array}{l}\text { A02:01 } \\
\text { C03:02 }\end{array}$ & 12 & 5 \\
\hline & & & B40:01 & & \\
\hline & V462E & 1 & B58:01 & 11 & 0 \\
\hline & & & C03:02 & & \\
\hline & D598Y & 1 & $\begin{array}{l}\text { A02:01 } \\
\text { B15:01 }\end{array}$ & 5 & 0 \\
\hline & A807V & 1 & $\begin{array}{l}\text { A02:01 } \\
\text { B40:01 } \\
\text { B58:01 } \\
\text { C03:02 }\end{array}$ & 13 & 6 \\
\hline \multirow{5}{*}{ GNAQ } & D95Y & 1 & $\begin{array}{l}\text { A24:10 } \\
\text { B18:02 } \\
\text { B39:01 } \\
\text { C07:02 }\end{array}$ & 8 & 0 \\
\hline & & & $\begin{array}{l}\text { A02:01 } \\
\text { A03:01 }\end{array}$ & & \\
\hline & T96S & 3 & A11:01 & 25 & 10 \\
\hline & & & B15:01 & & \\
\hline & & & B39:01 & & \\
\hline \multirow{7}{*}{$L R P 1 B$} & H4368Q & 1 & A11:02 & 1 & 0 \\
\hline & L1995M & 1 & C03:04 & 1 & 0 \\
\hline & R3026S & 1 & $\begin{array}{c}\text { A03:01 } \\
\text { B15:01 }\end{array}$ & 3 & 0 \\
\hline & & & A11:01 & & \\
\hline & R4062K & 1 & A33:03 & 8 & 0 \\
\hline & & & C03:02 & & \\
\hline & T2206I & 1 & A02:01 & 1 & 0 \\
\hline \multirow{5}{*}{ PIK3CA } & G106C & 1 & $\begin{array}{l}\text { A03:01 } \\
\text { A11:01 }\end{array}$ & 2 & 0 \\
\hline & H1047Y & 1 & B15:01 & 1 & 8 \\
\hline & & & A03:01 & & \\
\hline & V344M & 1 & A11:01 & 4 & 5 \\
\hline & & & $\mathrm{C} 14: 02$ & & \\
\hline \multirow{7}{*}{ PREX2 } & E1428K & 1 & A30:01 & 2 & 0 \\
\hline & H895Q & 1 & $\begin{array}{l}\text { B15:01 } \\
\text { C03:03 }\end{array}$ & 3 & 0 \\
\hline & Q102H & 1 & B15:01 & 1 & 0 \\
\hline & & & A02:01 & & \\
\hline & S1488L & 1 & A33:03 & 10 & 0 \\
\hline & & & B40:01 & & \\
\hline & & & C07:02 & & \\
\hline
\end{tabular}


PIK3CA, GNAQ, FAT4, and CDH1) that are recurrently mutated in GC. TP53, PIK3CA, and CDH1 are already reported to be mutated with high prevalence in GC according to TCGA and ACRG. FAT4 acts as a tumor suppressor by modulating Wnt/ß-catenin signaling pathway in GC [33]. Chen found that NRG1 was mutated in $10 \%$ of $78 \mathrm{GC}$ patients, and $8 \%$ of patients with mutations in BRCA2 were associated with longer survival [34]. Aberrant methylation of $L R P 1 B$ [35] and mutations on $L D L$ receptor-related protein $1 \mathrm{~B}$ cause SMAD4-induced GC growth [36]. PREX2 is involved in PIK3CA-PTEN-AKT signaling pathway. The frequency of GNAQ mutations was higher in intestinal-type gastric cancer [37]. Suppression of NOTCH1 signaling pathway could induce GC progression, drug resistance, and metastasis [38, 39]. The function of PCM1 and USP6 in gastric cancer remains exploitable.

Recurrent oncogenic mutations such as S37L and N289H in BRCA2, Q453L and A807V in FAT4, T96S in GNAQ, and H1047Y and V344M in PIK3CA were predicted to bind with HLA-A02:01, A03:01, A11:01, B15:01, B15:02, B58:01, B40:01, B39:01, and C03:02. Unlike the distribution percentage in African or Caucasian population, A02:01, A11:01, B58:01, B40:01, B15:01, and C03:02 HLA alleles are present in more than $5 \%$ of the Han Chinese population. The possibility to discover a common neoantigen target predicted by identical HLA allele and oncogenic mutation in Chinese patients is quite higher than African or Caucasian population.

While it is unknown why some individuals fail to respond favourably from T-cell-based neoantigen immunotherapy, targeting recurrent mutations of driver genes with HLA alleles still represents a promising avenue to treat eligible patients.

In this study, we analyzed the clinical features of Chinese GC patients and paired them with the somatic mutations and neoantigens present. We chose some reoccurring neoantigens and their associated oncogenes shared by several patients for the continued development of T-cell-based immunotherapy, such as vaccines. Due to the limited sample size of our study, we included the mutation frequencies from ICGC Project in our analysis to determine recurrent oncogenic mutations. Further studies should be conducted to confirm these results.

\section{Conclusions}

In conclusion, twelve recurrently mutated driver genes were identified in our study to further understand the mechanism of GC development. To identify the "druggable" targets, neoantigen profiling by TSNAD was done, highlighting several recurrent oncogenic driver mutations. Mutant peptides encoded by seven recurrent oncogenic mutations were predicted to bind with high frequency HLA alleles as tumorspecific neoantigens. These neoantigens are currently undergoing further experimental validation as potential targets for autologous T-cell immunotherapy to treat GC patients.

\section{Data Availability}

The data used to support the findings of this study are available from the corresponding author upon request.

\section{Ethical Approval}

Ethical approval was obtained from Research Ethics Committee of the First Affiliated Hospital, Zhejiang University School of Medicine, China. All experimental procedures were performed in accordance with the relevant guidelines (Approved Guidelines of the Clinical and Laboratory Standards Institute MM01-A3, MM13-A, and MM20-A).

\section{Consent}

The enrolment of human subjects in this study was done after informed consent forms were signed. Written consent for the collection and use of tissues for research purposes has been obtained.

\section{Conflicts of Interest}

The authors declare that there are no conflicts of interest regarding the publication of this paper.

\section{Authors' Contributions}

Jie Zhou and Wenyi Zhao contributed equally to this work.

\section{Acknowledgments}

The authors thank the patients and their families for their understanding and support of this research project. This work was supported by the National Key R\&D Program of China (2017YFC0908600), the Key Research and Development Program of Science and Technology, Department of Zhejiang Province (2018C03022), the National Natural Science Foundation of China (81403026 and 81430081), the Zhejiang Provincial Natural Science Foundation of China (LY17H310001), and Fundamental Research Funds for the Central University.

\section{Supplementary Materials}

Supplementary 1. Additional file 1: Table S1: the distribution of 40 recurrently mutated genes in 32 GC patients.

Supplementary 2. Additional file 2: Table S2: HLA typing of 32 gastric cancer patients.

Supplementary 3. Additional file 3: Table S3: the list of predicted neoantigens in 32 gastric cancer patients.

\section{References}

[1] A. R. Tan and S. M. Swain, "Ongoing adjuvant trials with trastuzumab in breast cancer," Seminars in Oncology, vol. 30, supplement 16, no. 5, pp. 54-64, 2003.

[2] N. Ferrara, K. J. Hillan, H.-P. Gerber, and W. Novotny, "Discovery and development of bevacizumab, an anti-VEGF antibody for treating cancer," Nature Reviews Drug Discovery, vol. 3, no. 5, pp. 391-400, 2004. 
[3] K. V. Foyil and N. L. Bartlett, "Anti-CD30 antibodies for Hodgkin lymphoma," Current Heart Failure Reports, vol. 5, no. 3, pp. 140-147, 2010.

[4] S. P. Treon, J. D. Soumerai, Z. R. Hunter et al., "Long-term follow-up of symptomatic patients with lymphoplasmacytic lymphoma/Waldenström macroglobulinemia treated with the anti-CD52 monoclonal antibody alemtuzumab," Blood, vol. 118, no. 2, pp. 276-281, 2011.

[5] R. A. Morgan, J. C. Yang, M. Kitano, M. E. Dudley, C. M. Laurencot, and S. A. Rosenberg, "Case report of a serious adverse event following the administration of $t$ cells transduced with a chimeric antigen receptor recognizing ERBB2," Molecular Therapy, vol. 18, no. 4, pp. 843-851, 2010.

[6] N. McGranahan, A. J. S. Furness, R. Rosenthal et al., "Clonal neoantigens elicit $\mathrm{T}$ cell immunoreactivity and sensitivity to immune checkpoint blockade," Science, vol. 351, no. 6280, pp. 1463-1470, 2016.

[7] M. Rajasagi, S. A. Shukla, E. F. Fritsch et al., "Systematic identification of personal tumor-specific neoantigens in chronic lymphocytic leukemia," Blood, vol. 124, no. 3, pp. 453-462, 2014.

[8] P. A. Ott, Z. Hu, D. B. Keskin et al., "An immunogenic personal neoantigen vaccine for patients with melanoma," Nature, vol. 547, no. 7662, pp. 217-221, 2017.

[9] E. Tran, S. Turcotte, A. Gros et al., "Cancer immunotherapy based on patient with epithelial cancer," Science, vol. 9, pp. 641646, 2014.

[10] R. L. Siegel, K. D. Miller, and A. Jemal, "Cancer statistics, 2015," CA: A Cancer Journal for Clinicians, vol. 69, no. 1, pp. 7-34, 2019.

[11] H. H. Hartgrink, E. P. Jansen, N. C. van Grieken, and C. J. van de Velde, "Gastric cancer," The Lancet, vol. 374, no. 9688, pp. 477-490, 2009.

[12] A. J. Bass, V. Thorsson, I. Shmulevich et al., "Comprehensive molecular characterization of gastric adenocarcinoma," Nature, vol. 513, no. 7517, pp. 202-209, 2014.

[13] R. Cristescu, J. Lee, M. Nebozhyn et al., "Molecular analysis of gastric cancer identifies subtypes associated with distinct clinical outcomes," Nature Medicine, vol. 21, no. 5, pp. 449-456, 2015.

[14] P. Charoentong, F. Finotello, M. Angelova et al., "Pan-cancer immunogenomic analyses reveal genotype-immunophenotype relationships and predictors of response to checkpoint blockade," Cell Reports, vol. 18, no. 1, pp. 248-262, 2017.

[15] R. J. Hartmaier, J. Charo, D. Fabrizio et al., "Genomic analysis of 63,220 tumors reveals insights into tumor uniqueness and targeted cancer immunotherapy strategies," Genome Medicine, vol. 9, no. 1, pp. 1-9, 2017.

[16] C. Chen, C. Shi, X. Huang et al., "Molecular profiles and mutation burden analysis in Chinese patients with gastric carcinoma," bioRxiv, 2018.

[17] J. Wu, W. Zhao, B. Zhou et al., "TSNAdb: a database for tumorspecific neoantigens from immunogenomics data analysis," Genomics, Proteomics \& Bioinformatics, vol. 16, no. 4, pp. 276282, 2018.

[18] Z. Zhou, X. Lyu, J. Wu et al., "TSNAD: an integrated software for cancer somatic mutation and tumour-specific neoantigen detection," Royal Society Open Science, vol. 4, no. 4, 2017.

[19] R. Li, C. Yu, Y. Li et al., "SOAP2: an improved ultrafast tool for short read alignment," Bioinformatics, vol. 25, no. 15, pp. 19661967, 2009.

[20] I. Hoof, B. Peters, J. Sidney et al., "NetMHCpan, a method for MHC class i binding prediction beyond humans," Immunogenetics, vol. 61, no. 1, pp. 1-13, 2009.
[21] W. L. Bi, N. F. Greenwald, M. Abedalthagafi et al., "Genomic landscape of high-grade meningiomas," npj Genomic Medicine, vol. 2, no. 1, pp. 1-14, 2017.

[22] L. B. Alexandrov, S. Nik-Zainal, D. C. Wedge et al., "Signatures of mutational processes in human cancer," Nature, vol. 500, no. 7463, pp. 415-421, 2013.

[23] L. B. Alexandrov, S. Nik-Zainal, D. C. Wedge, P. J. Campbell, and M. R. Stratton, "Deciphering signatures of mutational processes operative in human cancer," Cell Reports, vol. 3, no. 1, pp. 246$259,2013$.

[24] Y. Li, C. Wu, T. Chen et al., "Effects of RNAi-mediated MUC4 gene silencing on the proliferation and migration of human pancreatic carcinoma BxPC-3 cells," Oncology Reports, vol. 36, no. 6, pp. 3449-3455, 2016.

[25] J. S. Cho, M. H. Park, J. S. Lee, and J. H. Yoon, "Reduced MUC4 expression is a late event in breast carcinogenesis and is correlated with increased infiltration of immune cells as well as promoter hypermethylation in invasive breast carcinoma," Applied Immunohistochemistry \& Molecular Morphology, vol. 23, no. 1, pp. 44-53, 2015.

[26] H. Fu, Y. Liu, L. Xu et al., "Low expression of mucin-4 predicts poor prognosis in patients with clear-cell renal cell carcinoma," Medicine, vol. 95, no. 17, Article ID e3225, 2016.

[27] P.-A. Gourraud, P. Khankhanian, N. Cereb et al., "HLA diversity in the 1000 genomes dataset," PLoS ONE, vol. 9, no. 7, 2014.

[28] Q. J. Wang, Z. Yu, K. Griffith, K.-I. Hanada, N. P. Restifo, and J. C. Yang, "Identification of T-cell receptors targeting KRASmutated human tumors," Cancer Immunology Research, vol. 4, no. 3, pp. 204-214, 2016.

[29] E. Tran, P. F. Robbins, Y.-C. Lu et al., “T-cell transfer therapy targeting mutant KRAS in cancer," The New England Journal of Medicine, vol. 375, no. 23, pp. 2255-2262, 2016.

[30] M. H. Andersen, J. Fensterle, S. Ugurel et al., "Immunogenicity of constitutively active V599EBRaf," Cancer Research, vol. 64, no. 15, pp. 5456-5460, 2004.

[31] R. Somasundaram, R. Swoboda, L. Caputo et al., "Human leukocyte antigen-A2-restricted CTL responses to mutated BRAF peptides in melanoma patients," Cancer Research, vol. 66, no. 6, pp. 3287-3293, 2006.

[32] T. Yamada, K. Azuma, E. Muta et al., "EGFR T790M mutation as a possible target for immunotherapy; identification of HLA$\mathrm{A} * 0201$-restricted $\mathrm{T}$ cell epitopes derived from the EGFR T790M mutation," PLoS ONE, vol. 8, no. 11, pp. 3-12, 2013.

[33] J. Cai, D. Feng, L. Hu et al., "FAT4 functions as a tumour suppressor in gastric cancer by modulating Wnt $\beta$-catenin signalling," British Journal of Cancer, vol. 113, no. 12, pp. 17201729, 2015.

[34] K. Chen, D. Yang, X. Li et al., "Mutational landscape of gastric adenocarcinoma in Chinese: Implications for prognosis and therapy," Proceedings of the National Acadamy of Sciences of the United States of America, vol. 112, no. 4, pp. 1107-1112, 2015.

[35] D. H. Kim, Y. Nagano, I. Choi et al., "Aberrant methylation impairs low density lipoprotein receptor-related protein $1 \mathrm{~B}$ tumor suppressor function in gastric cancer," Genes Chromosomes Cancer, vol. 92, no. 49, pp. 412-424, 2010.

[36] H. Takeda, A. G. Rust, J. M. Ward, C. C. Yew, N. A. Jenkins, and N. G. Copeland, "Sleeping Beauty transposon mutagenesis identifies genes that cooperate with mutant Smad4 in gastric cancer development," Proceedings of the National Acadamy of Sciences of the United States of America, vol. 113, no. 14, pp. E2057-E2065, 2016. 
[37] S. Ge, B. Li, Y. Li et al., "Genomic alterations in advanced gastric cancer endoscopic biopsy samples using targeted nextgeneration sequencing," American Journal of Cancer Research, vol. 7, no. 7, pp. 1540-1553, 2017.

[38] M.-D. Zang, L. Hu, Z.-Y. Fan et al., "Luteolin suppresses gastric cancer progression by reversing epithelial-mesenchymal transition via suppression of the Notch signaling pathway," Journal of Translational Medicine, vol. 15, no. 1, article no. 52, pp. 1-11, 2017.

[39] W. Lian, L. Zhang, L. Yang, and W. Chen, "AP-2 $\alpha$ reverses vincristine-induced multidrug resistance of SGC7901 gastric cancer cells by inhibiting the Notch pathway," Apoptosis, vol. 22, no. 7, pp. 933-941, 2017. 


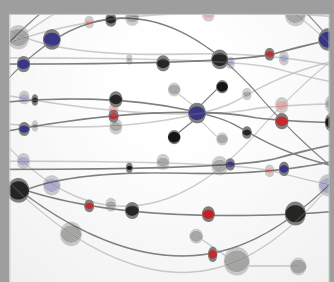

The Scientific World Journal
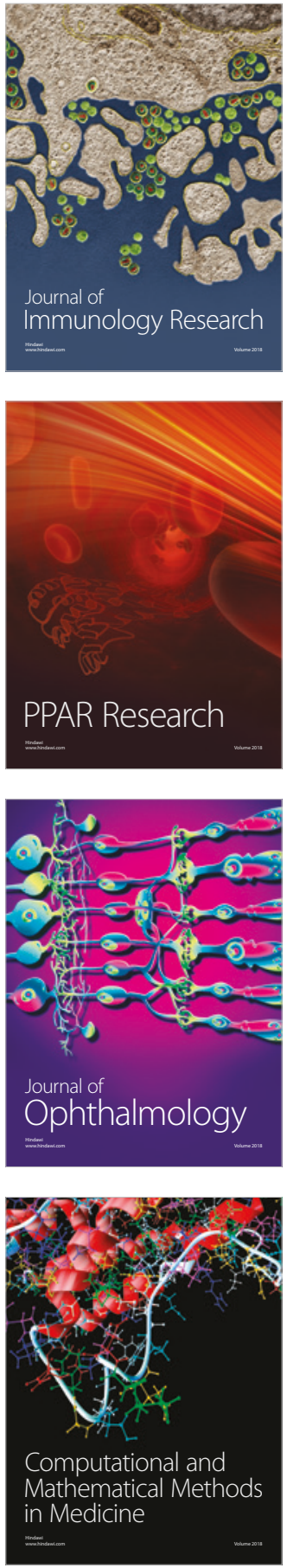

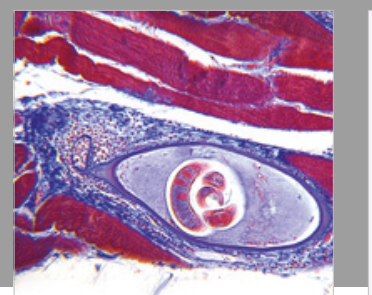

Gastroenterology Research and Practice

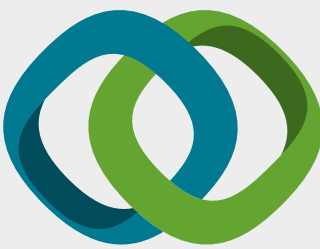

\section{Hindawi}

Submit your manuscripts at

www.hindawi.com
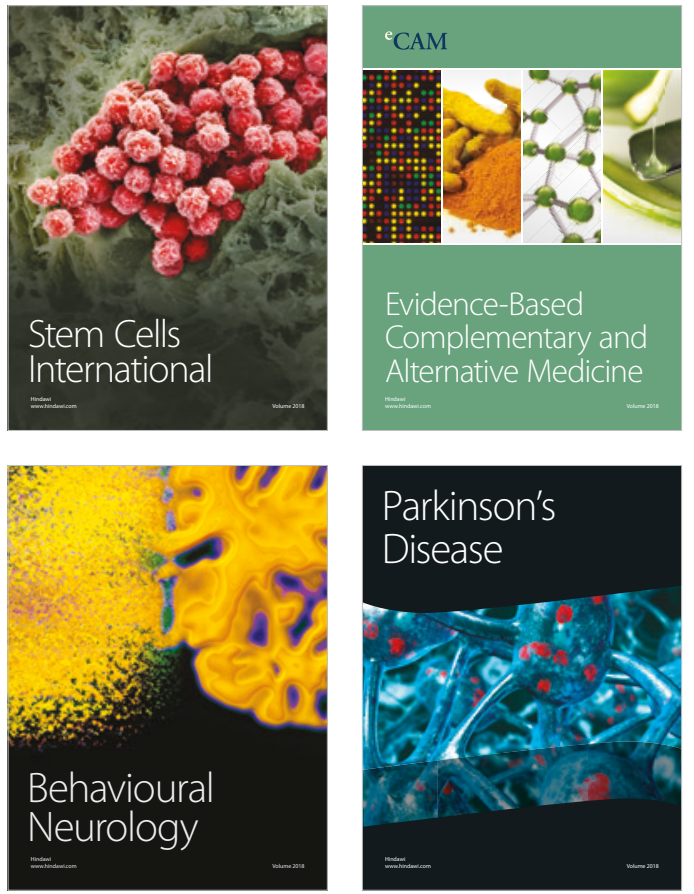

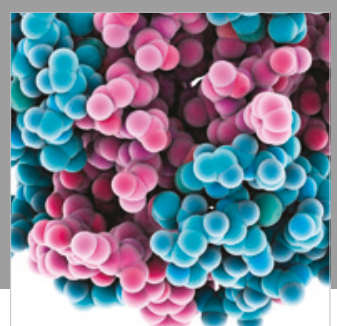

ournal of

Diabetes Research

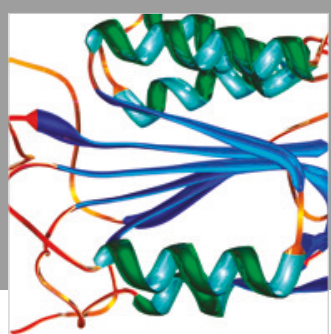

Disease Markers
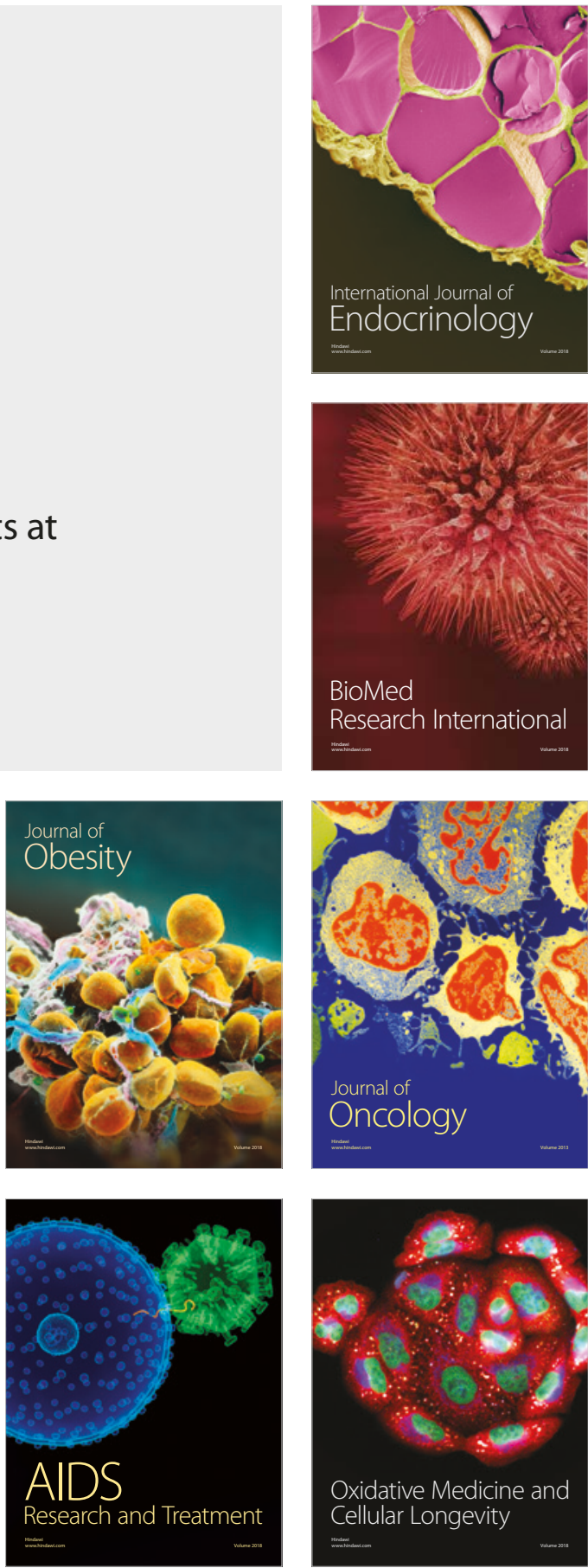